International Journal of Bifurcation and Chaos, Vol. 12, No. 5 (2002) 1159-1162

(c) World Scientific Publishing Company

\title{
ON OBSERVABILITY OF 3D CONTINUOUS-TIME AUTONOMOUS CHAOTIC SYSTEMS BASED ON SCALAR OUTPUT MEASUREMENT
}

\author{
XIAO-SONG YANG \\ Institute for Nonlinear Systems, Chongqing University of Posts and Telecomm., \\ Chongqing 400065, P.R. China \\ yangxs@cqupt.edu.cn
}

Received December 30, 2000; Revised October 1, 2001

\begin{abstract}
In this paper, by means of case studies we discuss an observable feature of 3D continuous-time autonomous chaotic systems through scalar output and its time derivatives. We observe that the Lorenz system, the Rössler system, the Chua circuit and the Chen system are all observable based on scalar output and its derivatives. This leads to our conjecture that chaotic motion described by $3 \mathrm{D}$ continuous-time autonomous dynamical system is observable based on a scalar output and its first- and second-order derivatives. Finally, we present some mathematical analysis and put up some theoretical questions for future studies.
\end{abstract}

Keywords: Observability; scalar output measurement; chaotic systems.

\section{Introduction}

It is common practice in applied chaotic systems studies to implement chaos control and chaos synchronization via a scalar signal (as a driving signal in coupled systems). A natural question thus arises: Is it always possible to utilize a scalar output signal in chaos control or chaos synchronization? From the viewpoint of control theory, one is naturally motivated to ask the following question:

Can a chaotic dynamical system be observable through a scalar output (and perhaps also its time derivatives)?

Clearly the answer to this question is of academic significance as well as practical importance.

A theory relevant to this topic is the well-known delay coordinate method [Takens, 1981], which is to construct an attractor via scalar measurement.

In this paper, we discuss the observability of chaotic systems in terms of output and its time derivatives, i.e. all the variables can be reconstructed via a scalar system output and its time derivatives. We will demonstrate such observability of chaotic dynamical systems by case studies of some typical chaotic systems in the literature. We will also present some mathematical analysis and discussion on this problem of interest.

\section{Case Studies}

The observabiliy problem in terms of reconstruction of all state variables by output and its time derivatives is not new in control theory, although here it is a little different from the standard definition of observability in control theory where time derivatives are generally not used. It is also quite well known in nonlinear dynamics theory [Eckmann \& Ruelle, 1985]. In this paper, however, we are concerned with an interesting problem that has not been well studied, namely, whether or not it is always possible to determine or reconstruct all state variables by using a scalar output and perhaps its $i$ th time derivatives for $i \leq n-1$, where $n$ is the dimension of the system under consideration. 
The following examples seem to suggest a positive answer to the above question, at least for $3 \mathrm{D}$ continuous-time autonomous chaotic dynamical systems. $1963]$

First, we consider the Lorenz system [Lorenz,

$$
\begin{aligned}
& \dot{x}=a(y-x) \\
& \dot{y}=c x-x z-y \\
& \dot{z}=x y-b z
\end{aligned}
$$

Let $h(x, y, z)=x$ be the output. Then

$$
\ddot{x}=a(\dot{y}-\dot{x})=a(c x-x z-y-a y+a x) .
$$

The variables $y$ and $z$ can be solved as follows:

$$
\begin{aligned}
& y=\frac{\dot{x}}{a}+x \\
& z=\left(\frac{\ddot{x}}{a}-a c x+a y+a^{2} y-a^{2} x\right) / x, \text { for } x \neq 0
\end{aligned}
$$

Note that the plane $x=0$ is not invariant under the flow of (1), and that any nontrivial orbit passing through the plane $x=0$ will go through this plane in discrete time. It can be seen that the variables $y$ and $z$ can be computed in terms of $x$ and its derivatives for "most" of the time (its precise meaning will be given in Definition 1 below).

For the Rössler system [Rössler, 1979]

$$
\begin{aligned}
& \dot{x}=-y-z \\
& \dot{y}=x+a y \\
& \dot{z}=x z-b z+c
\end{aligned}
$$

Take $h(x, y, z)=x$ to be the output. Then

$$
\begin{aligned}
& \dot{x}=-y-z \\
& \ddot{x}=-x-a y-x z+b z-c
\end{aligned}
$$

From

$$
\left|\begin{array}{cc}
-1 & -1 \\
-a & -(x-b)
\end{array}\right|=x-a-b
$$

it follows that $y$ and $z$ can be solved in a region off the plane $x=a+b$.

Note, however, that the chaotic attractor cannot be contained within the plane $x=a+b$. But the variables $y$ and $z$ again can be computed in terms of $x$ and its derivatives for "most" of the time (its precise meaning will be given in Definition 1 below).
As for the chaotic Chua's circuit [Chua et al., $1986]$

$$
\begin{aligned}
& \dot{x}=\alpha(x-y-f(x)) \\
& \dot{y}=x-y+z \\
& \dot{z}=-\beta y
\end{aligned}
$$

we may take the variable $z$ as the output. In this case, it is easy to obtain

$$
\begin{aligned}
& y=-\frac{\dot{z}}{\beta} \\
& x=-\frac{\ddot{z}}{\beta}-\frac{\dot{z}}{\beta}-z
\end{aligned}
$$

The variables $y$ and $x$ can be computed in terms of $z$ and its derivatives for all the time.

The same arguments also apply to Chen's system [Chen \& Ueta, 1999]

$$
\begin{aligned}
& \dot{x}=a(y-x) \\
& \dot{y}=(c-a) x-x z+c y \\
& \dot{z}=x y-b z
\end{aligned}
$$

From all the above examples, it seems to be justifiable to conjecture that chaotic attractors can be observed, hence be constructed, via a scalar output and its first- and second-order derivatives for $3 \mathrm{D}$ autonomous systems of ordinary differential equations.

\section{A General Discussion}

Some notions and results are first presented (for details, see [Nijmeijer \& van der Schaft, 1990]).

Consider the following system with a scalar output:

$$
\begin{aligned}
& \dot{x}=F(x), \quad x \in R^{n} \\
& y=h(x)
\end{aligned}
$$

Let $k(x)$ be a scalar differentiable function and $v(x)$ be a vector field. The Lie derivative of $k$ along $v$ is defined to be

$$
L_{v} k=\langle v(x), \operatorname{grad} k(x)\rangle,
$$

where $\langle$,$\rangle is the Euclidean inner product. The i$ th Lie derivative of $k$ along $v$ is defined inductively as follows:

$$
L_{v}^{i} k=\left\langle v(x), \operatorname{grad} L_{v}^{i-1} k(x)\right\rangle
$$

It is easy to obtain the following result. 
Theorem 1. If the map

$$
\Phi=\left(h(x), L_{F} h(x), \ldots, L_{F}^{n-1} h(x)\right)^{T}
$$

is a diffeomorphism, then (1) is observable through output $y(t)$ and its ith derivatives for $i \leq n-1$. In addition, one has $x(t)=$ $\Phi^{-1}\left(y(t), y(t), \ldots, y^{(n-1)}(t)\right)^{T}$.

Remark 1. It is easy to verify, by direct computation, that the $i$ th derivative of $y(t)$ is $L_{F}^{i} h(x)$. In addition, to assure the observability for (1), it is sufficient for the map $\Phi$ to be diffeomorphic on the attractor of (1).

Let $O$ be the linear space of function $h(x)$ and all its repeated Lie derivatives, $L_{F}^{i} h, i=0,1, \ldots$. Let

$$
D O=\operatorname{span}\{d H: H \in O\}
$$

Then, it is easy to see that the system is locally observable in a neighborhood of $\bar{x}$ if $\operatorname{dim} D O=n$ at the point $\bar{x}$.

Remark 2. From the theory of reconstruction of attractors [Eckmann \& Ruelle, 1985], it is easy to see that if $i$ is large enough then generically one is able to reconstruct the dynamics of the attractor by using a scalar output and its derivatives. However, what we are concerned with here is whether or not it is always true that a $3 \mathrm{D}$ continuous-time autonomous chaotic dynamical system is observable by using a scalar output and its $i$ th derivatives for $i \leq 2$. This can be dealt with within the context of nonlinear control theory.

For further discussion, we need the following concept.

Definition 1. Consider an output $y(t)=h(x(t))$ of (5). Let $T=\left[t_{1}, t_{2}\right)$ be a time interval and $K \subset T$ be a subset of zero measure (for instance, in the Lebesgue measure). If the state variables $x(t)$ can be reconstructed using $y(t)$ and its $i$ th time derivatives for $t \in T-K, i=1,2$, then (5) is said to be almost observable during the period $T=\left[t_{1}, t_{2}\right)$. In other words, (5) is observable "most" of the time within the time interval $T=\left[t_{1}, t_{2}\right)$.

For 3D continuous-time dynamical systems, we can make a plausible assertion that chaos can be observable, at least locally, based on a scalar output and its time derivatives "most" of the time.
To this end, let us consider the following system in a general form:

$$
\dot{x}=F(x), \quad x \in R^{3}
$$

For convenience, we assume the smoothness of (6). Let $A$ be a chaotic attractor of (1) and take $x_{1}$ to be the scalar output of the system. Then, it is easy to see that $L_{F} x_{1}$ and $L_{F}^{2} x_{1}$ cannot vanish on the attractor, because chaotic attractor cannot be located on a 2D manifold for the autonomous case. Furthermore, it can be proved that the chaotic attractor is not contained in any singularity set, denoted by $\Sigma(\Phi)$, where

$$
\Phi=\left(x_{1}, L_{F} x_{1}, L_{F}^{2} x_{1}\right)^{T} .
$$

To develop a rigorous argument, let us recall some concept from singularity theory [Golubitsky \& Guillermin, 1973].

Definition 2. Consider a smooth map $\Phi: R^{n} \rightarrow$ $R^{n}$. A point $x$ is said to be a critical point of a smooth map $\Phi$, if the Jacobian matrix $D \Phi$ is degenerate at $x$. The point $x$ is said to be in class $\Sigma^{i}$ for $\Phi$, if the dimension of the kernel of $D \Phi$ at $x$ is equal to $i$. All the points of class $\Sigma^{i}$ for $\Phi$ form a subset $\Sigma^{i}(\Phi)$ of the space $R^{n}$.

A well-known result about the set $\Sigma^{i}(\Phi)$ in singularity theory is the following.

Proposition 3. If the 1-jet $j^{1} \Phi$ of the map $\Phi$ transversely intersects with $S_{i}$, then $\Sigma^{i}(\Phi)$ is a smooth submanifold and the dimension of $\Sigma^{i}(\Phi)$ is $n-i^{2}$. Therefore, generically, $\Sigma^{i}(\Phi)$ is a smooth submanifold and the dimension of $\Sigma^{i}(\Phi)$ is $n-i^{2}$. Here, $S_{i}=\left\{\Sigma \in J^{1}(X, X) \mid \operatorname{corank} \sigma=i\right\}$ is a submanifold of the $i$-jet $J^{1}(X, X)$.

It is easy to see that the following statement holds true.

Proposition 4. For the map considered in (7), one has

$$
\operatorname{dim} \Sigma^{i}(\Phi)<3 .
$$

Generically, only the situation with $i \leq 1$ is possible.

Remark 3. Proposition 4 means that the set of critical points of $\Phi$ has zero measure and the chaotic attractor is not contained in $\Sigma^{i}(\Phi)$ if the map transversely intersects with $S_{1}$ consequently (which is 
generically true), the chaotic attractor should be generically observable locally "most" of the time.

For chaotic systems, the following question is interesting:

Suppose that $\Phi$ gives rise to a chaotic attractor. Does $J^{1} \Phi$ transversely intersect with $S_{i}$ in the vicinity of the attractor?

If this is the case, then we can be sure that a $3 \mathrm{D}$ continuous-time chaotic dynamical system will be at least locally observable through a scalar output (and its first- and second-order time derivatives) "most" of the time. But we do not have an answer for the above question at the moment.

\section{Conclusion}

In this paper, we have presented some case studies for $3 \mathrm{D}$ continuous-time autonomous chaotic systems and provided a mathematical argument to support the conjecture that a chaotic attractor can be observable via a scalar output and its (first- and second-order) time derivatives. Further theoretical investigations still remain to be carried out in the near future.

\section{Acknowledgments}

This work is partially supported by the Applied Science Foundation of Chongqing City Scientific Community. The author wishes to thank the referee for the useful comments and particularly Prof. G. Chen for helpful discussions and suggestions.

\section{References}

Chen, G. \& Ueta, T. [1999] "Yet another chaotic attractor," Int. J. Bifurcation and Chaos 7, 1465-1466.

Chua, L. O., Komuro, M. \& Matsumato, T. [1986] "The double scroll family," IEEE Trans. Circuits Syst. CAS-33, 1073-1118.

Eckmann, J.-P. \& Ruelle, D. [1985] "Ergodic theory of chaos and strange attractors," Rev. Mod. Phys. 57, 617-656.

Golubitsky, M. \& Guillermin, V. W. [1973] Stable Mappings and Their Singularities (Springer-Verlag, NY).

Lorenz, E. N. [1963] "Deterministic nonperiodic flow," J. Atmos. Sci. 20, 130-141.

Nijmeijer, H. \& van der Schaft, A. [1990] Nonlinear Dynamical Control Systems (Springer-Verlag, NY).

Rössler, O. E. [1979] "Continuous chaos: Four prototype equations," Ann. N. Y. Acad. Sci. 316, 376-392.

Takens, F. [1981] Dynamical Systems and Turbulence (Springer-Verlag, NY). 